hep-th/9801135

UT-Komaba/98-1

OU-HET 289

\title{
Fermionic Zero Mode and String Creation between D4-Branes at Angles
}

\author{
Takuhiro Kitao \\ Institute of Physics, University of Tokyo, Komaba, Tokyo 153, Japan \\ and \\ Nobuyoshi Ohta" and Jian-Ge Zhouf \\ Department of Physics, Osaka University, Toyonaka, Osaka 560, Japan
}

\begin{abstract}
We study the creation of a fundamental string between D4-branes at angles in string theory. It is shown that $\mathrm{R}(-1)^{\mathrm{F}}$ part of the one-loop potential of open string changes its sign due to the change of fermionic zero-mode vacua when the branes cross each other. As a result the effective potential is independent of the angles when supersymmetry is partially unbroken, and leads to a consistent picture that a fundamental string is created in the process. We also discuss the s-rule in the configuration. The same result is obtained from the one-loop potential for the orthogonal D4-branes with non-zero field strength. The result is also confirmed from the tension obtained by deforming the Chern-Simons term on one D4-brane, which is induced by another tilted D4-brane.
\end{abstract}

\footnotetext{
*e-mail address: kitao@hep1.c.u-tokyo.ac.jp

${ }^{\dagger}$ e-mail address: ohta@phys.wani.osaka-u.ac.jp

${ }_{\ddagger}^{\ddagger}$ e-mail address: jgzhou@phys.wani.osaka-u.ac.jp, JSPS postdoctral fellow
} 
Recently it has been pointed out from various points of view that a brane (string) is created when certain two branes cross each other [1]-[14]. This is originally suggested in the field theory analysis on D-branes in ref. [1]. Consistencies of this result are confirmed by deforming the Chern-Simons term of the system [3] and by the one-loop potentials of open strings [4. Moreover, the results of M(atrix) theory also support this fact [6, \&] and the authors of refs. 110, 11, 12 have explained the brane creation by using the technique of holomorphic embedding of M5-brane or M2-brane into Taub-NUT space induced by Kaluza-Klein monopole.

In this paper, we study the creation of a fundamental string between two D4-branes at angles, in which the configuration has less supercharges than those of orthogonal case [15]-22]. This system was examined in the framework of the M(atrix) theory in ref. [8], and we intend to study it and its variant in string theory in more detail. Of course, we expect that the result is the same as that of $\mathrm{M}$ (atrix) theory [8] because the approach of M(atrix) theory necessarily involves the theory of bound states of D-branes whose Tduality is the theory of branes with angles discussed in ref. [20]. However, we find that there is a subtle issue which needs clarification.

To discuss the string creation, we use the one-loop potential due to the open strings between the branes at angles. Amplitudes for such system have been obtained in ref. [21] to determine the condition of unbroken supersymmetry, and we can simply read off the potential from that work. We also obtain the one-loop potential from open strings between the orthogonal branes with condensation which is similar to the case with angles. When supersymmetry is partially unbroken, these potentials vanish. The problem is what happens when one brane adiabatically crosses another brane, keeping the partially unbroken supersymmetry. The authors of ref. [4 have interpreted this situation as the system of brane-anti-brane, and obtained the result which is consistent with the creation of a fundamental string, that is, the effective potential of the string tension times the distance between the branes, which is canceled by a string created between these branes. They discussed the case that D0-brane passes through D8-brane, in which the $\mathrm{NS}(-1)^{\mathrm{F}}$ term vanishes because of the fermionic zero modes.

On the other hand, in the case with angles, $\mathrm{NS}(-1)^{\mathrm{F}}$ term gives generally non-zero contribution which depends on angles. As a consequence, it turns out that if we interpreted 
the system as that of brane-anti-brane when one brane adiabatically crossed another, the potential would be dependent on the angles. This is a very strange result if we try to understand it in terms of a string stretched between the two branes. We find that in our present situation only the $\mathrm{R}(-1)^{\mathrm{F}}$ term changes the sign after crossing because the vacua defined by fermionic zero mode change. The result is then independent of the angles and is given by the string tension times the distance between the branes, allowing the physical interpretation in terms of string creation. The potential is canceled by the contribution from the string created between the branes.

In the case of orthogonal two D-branes, it has been pointed out in ref. [3] that the force induced by NS and R terms which is one half of the string tension 23] cancels the tension induced by Chern-Simons term. We also calculate this tension from the Chern-Simons term in the case with angles. We find that the result is independent of the angles and is equal to one half of string tension, the same as that found in ref. [3]. This confirms our result that the effective potential is independent of the angles and only the $\mathrm{R}(-1)^{\mathrm{F}}$ term changes the sign after brane crossing.

Our results also indicate that the s-rule of Hanany and Witten is valid [1]. The rule translated into our present setting states that a configuration with more than one fundamental string joining the two D4-branes cannot be supersymmetric. As we will see, this rule can be understood as the uniqueness of the chiral fermionic zero-mode vacuum.

Let us start with the configuration of two D4-branes, one of which has tilted worldvolume to that of another D4-brane. The boundary conditions of the open string on one D4-brane (denoted as D4) are

$$
\begin{aligned}
& \partial_{\sigma} X^{\mu}=0, \quad \mu=0, \ldots, 4, \\
& X^{\mu}=0, \quad \mu=5, \ldots, 9,
\end{aligned}
$$

at $\sigma=0$, where $\sigma$ is the world-sheet coordinate which spans $[0, \pi]$. The boundary conditions for fermions follow from the world-sheet supersymmetry

$$
\delta X^{\mu}=\bar{\epsilon} \psi^{\mu}
$$

Those at $\sigma=\pi$ on another D4-brane (denoted as D4') are

$$
\partial_{\sigma} X^{0}=0
$$




$$
\begin{aligned}
& \partial_{\sigma} X^{i} \cos \left(\theta_{i} \pi\right)-\partial_{\sigma} X^{i+4} \sin \left(\theta_{i} \pi\right)=0, \\
& X^{i} \sin \left(\theta_{i} \pi\right)+X^{i+4} \cos \left(\theta_{i} \pi\right)=0, \quad i=1, \ldots, 4, \\
& X^{9}=b
\end{aligned}
$$

where $b$ is the distance between the two branes and $\left\{\theta_{i} \pi\right\}$ are the angles parameterizing our two D4-brane configuration.

The one-loop amplitude of the open string which satisfies the above boundary conditions has been obtained in ref. [21]. Assuming that all the four angles are non-zero, 1] we find that the potential between these D4-branes is

$$
\begin{gathered}
V=-\int_{0}^{\infty} \frac{d t}{2 t} \frac{\mathrm{e}^{-\frac{b^{2} t}{2 \pi \alpha^{\prime}}}}{\left(8 \pi^{2} \alpha^{\prime} t\right)^{\frac{1}{2}}}\left[-\prod_{k=1}^{4} \frac{\Theta_{2}\left(i \theta_{k} t \mid i t\right)}{\Theta_{1}\left(i \theta_{k} t \mid i t\right)}+\prod_{k=1}^{4} \frac{\Theta_{3}\left(i \theta_{k} t \mid i t\right)}{\Theta_{1}\left(i \theta_{k} t \mid i t\right)}\right. \\
\left.+\prod_{k=1}^{4} \frac{\Theta_{1}\left(i \theta_{k} t \mid i t\right)}{\Theta_{1}\left(i \theta_{k} t \mid i t\right)}-\prod_{k=1}^{4} \frac{\Theta_{4}\left(i \theta_{k} t \mid i t\right)}{\Theta_{1}\left(i \theta_{k} t \mid i t\right)}\right] .
\end{gathered}
$$

The terms in the bracket are the contributions from R, NS, R $(-1)^{\mathrm{F}}$ and $\mathrm{NS}(-1)^{\mathrm{F}}$ sectors, respectively. The author of ref. [21] has derived the condition that the amplitude vanishes, in the search for the criterion that supersymmetry is partially unbroken. It is given by

$$
\theta_{1} \pm \theta_{2} \pm \theta_{3} \pm \theta_{4}=0, \quad \bmod 2,
$$

for which we have

$$
\prod_{k=1}^{4} \Theta_{1}\left(i \theta_{k} t \mid i t\right)-\prod_{k=1}^{4} \Theta_{2}\left(i \theta_{k} t \mid i t\right)+\prod_{k=1}^{4} \Theta_{3}\left(i \theta_{k} t \mid i t\right)-\prod_{k=1}^{4} \Theta_{4}\left(i \theta_{k} t \mid i t\right)=0 .
$$

Indeed, eq. (5) is the same as that derived in supergravity in ref. [22], in which it has been shown that there is $1 / 16$ unbroken supersymmetry if eq. (5) is satisfied. In what follows, we will mainly concentrate on the case (5).

Let us consider what happens when the above one brane passes through another adiabatically in the direction of $x^{9}$ from $x^{9}=|b|$ to $x^{9}=-|b|$. We can regard this situation as the sum of two parts. One is that of one of the branes hooked by another

\footnotetext{
${ }^{1}$ For zero angle $\theta_{i}$, it is easy to do a similar calculation. One finds that the summation of the bosonic degrees of freedom is replaced by the product of the integral over the momentum $p_{i}$ and the volume of the direction $X^{i}$. It should be possible to continuously interpolate the zero angle limit in eq. (4), but it seems complicated to do so explicitly.
} 
brane, which is expected to be a fundamental string. Another part is that from the two branes passing by without hooking. If the interaction between the two branes of the latter part in this situation is the string tension times the distance between the branes, the first part is expected to represent the created fundamental string to cancel this interaction between the two branes.

What is the difference between the state before crossing ("configuration [A]") in which $1 / 16$ SUSY is unbroken and that after crossing ("configuration [B]") ignoring the hooking part? The only difference is the vacua defined by the R-fermion zero modes, which exist only in the world-sheet fermions $\psi^{0}$ and $\psi^{9}$. There are no other zero modes because the boundary conditions with the angles shift the R-fermion zero modes of the rotated directions. We define the vacua $| \pm\rangle$ by

$$
\left(\psi_{0}^{0} \pm \psi_{0}^{9}\right)| \pm\rangle=0 .
$$

By GSO projection, either + or - is projected out, so that only one space-time massless chiral fermion can exist when the two branes intersect. Configuration $[\mathrm{A}]$ is related to $[\mathrm{B}]$ by the parity transformation in the direction of $x^{9}$. In order to preserve the supersymmetry (2), $\psi_{0}^{9}$ transforms into $-\psi_{0}^{9}$ when the configuration changes from [A] to [B]. It follows that the vacuum of $[\mathrm{A}]$ is different from that of $[\mathrm{B}]$. In other words, the definition of GSO projection for the $\mathrm{R}$-sector is different between $[\mathrm{A}]$ and $[\mathrm{B}]$. As a result, the potential of $[B]$ is different from $[A]$ by the sign of the $R(-1)^{\mathrm{F}}$ term. By using the condition of unbroken supersymmetry (6), we find

$$
\begin{aligned}
V=-\frac{1}{2} \int_{0}^{\infty} \frac{d t}{t} & \frac{\mathrm{e}^{-\frac{b^{2} t}{2 \pi \alpha^{\prime}}}}{\left(8 \pi^{2} \alpha^{\prime} t\right)^{\frac{1}{2}}}\left[-\prod_{k=1}^{4} \frac{\Theta_{2}\left(i \theta_{k} t \mid i t\right)}{\Theta_{1}\left(i \theta_{k} t \mid i t\right)}+\prod_{k=1}^{4} \frac{\Theta_{3}\left(i \theta_{k} t \mid i t\right)}{\Theta_{1}\left(i \theta_{k} t \mid i t\right)}\right. \\
& \left. \pm \prod_{k=1}^{4} \frac{\Theta_{1}\left(i \theta_{k} t \mid i t\right)}{\Theta_{1}\left(i \theta_{k} t \mid i t\right)}-\prod_{k=1}^{4} \frac{\Theta_{4}\left(i \theta_{k} t \mid i t\right)}{\Theta_{1}\left(i \theta_{k} t \mid i t\right)}\right] \\
= & -\frac{1}{2} \int_{0}^{\infty} \frac{d t}{t} \frac{\mathrm{e}^{-\frac{b^{2} t}{2 \pi \alpha^{\prime}}}}{\left(8 \pi^{2} \alpha^{\prime} t\right)^{\frac{1}{2}}}(-1 \pm 1) \\
= & -\frac{T_{0}}{2}(1 \mp 1)|b|,
\end{aligned}
$$

where $-(+)$ in the last equation is for the configuration $[\mathrm{A}]([\mathrm{B}])$, and $T_{0}$ is the string tension $\frac{1}{2 \pi \alpha^{\prime}}$. This change of the sign occurs when the two branes intersect each other at

\footnotetext{
${ }^{2}$ More concrete picture of this "hooking" will be explained below.
} 
$b=0$. We see that the potential computed in eq. (8) for $[\mathrm{B}]$ is independent of the angles and equals the string tension times the distance. On the other hand, since we move one brane across another from $x^{9}=|b|$ to $x^{9}=-|b|$ adiabatically, the fermionic zero-mode vacuum is changed after crossing from $x^{9}=0$ all the way down to $x^{9}=-|b|$ and we get contributions from that part which exactly cancel the above potential. This is what we have called "hooking" in the above. Physically this should be understood as due to a string created between the two branes when supersymmetry is unbroken. In this way, the BPS property of the system is preserved before and after the brane crossing.

It is crucial that only the $\mathrm{R}(-1)^{\mathrm{F}}$ term changes its sign; if we interpreted the final configuration as brane-anti-brane system, both $\mathrm{NS}(-1)^{\mathrm{F}}$ and $\mathrm{R}(-1)^{\mathrm{F}}$ terms (related by modular transformation to the RR sector in the closed string channel) would change the signs, resulting in a potential dependent on the angles. Such potential could not be canceled by string tension, and would allow no physical interpretation. [

The fact that there is only one space-time massless chiral fermion exists at the intersection is closely related to the s-rule [1, 12, 14]. Our results indicate that only a single string is stretched between two D4-branes after crossing. By a chain of dualities, the special orthogonal case can be transformed to the configuration in which a D3-brane is suspended between an NS 5-brane and a D5-brane considered in ref. [1]. Thus our results give the generalization of this rule to more general angles.

It is easy to repeat the calculation when any one of the angles $\theta_{i}$ is zero under the condition (5), and we find that the potential vanishes and no string is created.

We can derive the same result in the case of orthogonal D4-D4' system with bound state [24]-29]. The boundary conditions of the open string on one D4-brane are

$$
\begin{aligned}
& \partial_{\sigma} X^{0}=0, \\
& \partial_{\sigma} X^{2 k-1}+2 \pi \alpha^{\prime} F_{(k)} \partial_{\tau} X^{2 k}=0, \\
& \partial_{\sigma} X^{2 k}-2 \pi \alpha^{\prime} F_{(k)} \partial_{\tau} X^{2 k-1}=0, \quad k=1, \quad 2, \\
& X^{\mu}=0, \quad \mu=5, \ldots 9,
\end{aligned}
$$

at $\sigma=0$, where $\tau$ is the world-sheet coordinate along with $\sigma$ and $F_{(k)}$ is the condensation

\footnotetext{
${ }^{3}$ In the absence of $\mathrm{NS}(-1)^{\mathrm{F}}$ term, these pictures make no difference, and both give the same consistent results for string creation 淉.
} 
of the field strength. The boundary conditions at $\sigma=\pi$ on another D4'-brane are

$$
\begin{aligned}
& \partial_{\sigma} X^{0}=0 \\
& X^{\mu}=0, \quad \mu=1, \ldots, 4, \\
& \partial_{\sigma} X^{2 k-1}+2 \pi \alpha^{\prime} F_{(k)} \partial_{\tau} X^{2 k}=0, \\
& \partial_{\sigma} X^{2 k}-2 \pi \alpha^{\prime} F_{(k)} \partial_{\tau} X^{2 k-1}=0, \quad k=3, \quad 4, \\
& X^{9}=b .
\end{aligned}
$$

By repeating the calculation similar to the above, we obtain the potential

$$
\begin{gathered}
V=-\int_{0}^{\infty} \frac{d t}{2 t} \frac{\mathrm{e}^{-\frac{b^{2} t}{2 \pi \alpha^{\prime}}}}{\left(8 \pi^{2} \alpha^{\prime} t\right)^{\frac{1}{2}}}\left[-\prod_{k=1}^{4} \frac{\Theta_{3}\left(i \epsilon_{k} t \mid i t\right)}{\Theta_{4}\left(i \epsilon_{k} t \mid i t\right)}+\prod_{k=1}^{4} \frac{\Theta_{2}\left(i \epsilon_{k} t \mid i t\right)}{\Theta_{4}\left(i \epsilon_{k} t \mid i t\right)}\right. \\
\left. \pm \prod_{k=1}^{4} \frac{\Theta_{4}\left(i \epsilon_{k} t \mid i t\right)}{\Theta_{4}\left(i \epsilon_{k} t \mid i t\right)}-\prod_{k=1}^{4} \frac{\Theta_{1}\left(i \epsilon_{k} t \mid i t\right)}{\Theta_{4}\left(i \epsilon_{k} t \mid i t\right)}\right]
\end{gathered}
$$

where $\epsilon_{k} \equiv \frac{\tan ^{-1}\left(2 \pi \alpha^{\prime} F_{(k)}\right)}{\pi}$ and \pm corresponds to the difference in the definitions of GSO projection. The condition for $1 / 16$ unbroken SUSY is the same as the case with angles:

$$
\epsilon_{1} \pm \epsilon_{2} \pm \epsilon_{3} \pm \epsilon_{4}=0, \quad \bmod 2 .
$$

With the help of eq. (6), the potential (11) is cast into

$$
V=-\int_{0}^{\infty} \frac{d t}{2 t} \frac{\mathrm{e}^{-\frac{b^{2} t}{2 \pi \alpha^{\prime}}}}{\left(8 \pi^{2} \alpha^{\prime} t\right)^{\frac{1}{2}}}(-1 \pm 1)=-\frac{T_{0}}{2}(1 \mp 1)|b| .
$$

This also shows that a fundamental string is created.

As a further check of our results, let us calculate the induced tension from the ChernSimons term. We again deal with the system of two D4-branes at angles. Following ref. [3] in which the system of D0 and D8-branes is discussed, we consider the Chern-Simons term on the first D4:

$$
\frac{\mu_{\mathrm{D} 4}}{4 !} \int d^{5} x \epsilon_{\nu_{0} \nu_{1} \nu_{2} \nu_{3} \nu_{4}} F_{(4)}^{\nu_{0} \nu_{1} \nu_{2} \nu_{3}} A^{\nu_{4}}
$$

where $F_{(4)} \equiv d C_{(3)}, C_{(3)}$ is the R-R 3-form gauge field, and $A^{\nu}$ is the $\mathrm{U}(1)$ gauge field on D4. The indices $\left\{\nu_{k}\right\}$ are those of the coordinates of the world-volume of the first D4, $x^{0,1, \ldots, 4}$. The second D4'-brane is the source of $F_{(4)}=* F_{(6)}$ in the integral (14). Assuming 
that $F_{(4)}$ depends on only $x^{1}, \ldots, x^{4}$ and that $A^{\nu}$ depends on only $x^{0}$, the above term reduces to

$$
\mu_{\mathrm{D} 4} \int\left(\prod_{i=1}^{4} d x_{i}\right) F_{(4)}^{1234} \int d s \frac{d x_{0}}{d s} A^{0}\left(x_{0}\right),
$$

where $s$ is a parameter on the D4 and can be supposed to be a world-sheet coordinate, and then $\mu_{\mathrm{D} 4} \int\left(\prod_{i=1}^{4} d x_{i}\right) F_{(4)}^{1234}$ is interpreted as the tension to be evaluated below.

On the other hand, the R-R charge $\mu_{\mathrm{D} 4^{\prime}}$ of $\mathrm{D} 4^{\prime}$ is

$$
\mu_{\mathrm{D} 4^{\prime}}=\int_{S_{4}} * F_{(6)} d^{4} x=\int_{S_{4}} F_{(4)} d^{4} x
$$

where $S_{4}$ is the 4 -sphere surrounding $\mathrm{D}^{\prime}$. Hence $\mathrm{F}_{(4)}$ can be taken as

$$
\frac{1}{4 !} \epsilon_{\nu_{0}^{\prime} \nu_{1}^{\prime} \nu_{2}^{\prime} \nu_{3}^{\prime} \nu_{4}^{\prime}} F_{(4)}^{\nu_{0}^{\prime} \nu_{1}^{\prime} \nu_{2}^{\prime} \nu_{3}^{\prime}}=\frac{\mu_{\mathrm{D} 4^{\prime}}}{r^{4} \Omega_{4}} \frac{x_{\nu_{4}^{\prime}}}{r}
$$

where all $\nu^{\prime}$ s are the indices of the directions which are orthogonal to the world-volume of $\mathrm{D}^{\prime}$, and $r$ and $\Omega_{4}$ are the radius of $S_{4}$ and the volume of a unit 4-sphere, respectively. We denote the directions of the world-volume of $\mathrm{D} 4^{\prime}$ as $x_{0}^{\prime}, \ldots, x_{4}^{\prime}$. Then the non-zero components of $F_{(4)}^{\nu_{0}^{\prime} \nu_{1}^{\prime} \nu_{2}^{\prime} \nu_{3}^{\prime}}$ are those with indices outside these dimensions:

$$
F_{(4)}^{5^{\prime} 6^{\prime} 7^{\prime} 8^{\prime}}, \quad F_{(4)}^{6^{\prime} 7^{\prime} 8^{\prime} 9^{\prime}}, \quad F_{(4)}^{7^{\prime} 8^{\prime} 9^{\prime} 5^{\prime}}, \quad F_{(4)}^{8^{\prime} 9^{\prime} 5^{\prime} 6^{\prime}}, \quad F_{(4)}^{9^{\prime} 5^{\prime} 6^{\prime} 7^{\prime}}
$$

To evaluate the contribution to $F_{(4)}^{1234}$ at a point $\mathrm{Q}$ on $\mathrm{D} 4$ from a point $\mathrm{P}$ on the second $\mathrm{D} 4^{\prime}$, let us consider the 5-dimensional plane which intersects $\mathrm{D} 4^{\prime}$ orthogonally at $\mathrm{P}$, and also D4 at $\mathrm{Q}$. The relation between the coordinates $\left\{x_{i}\right\}$ for $\mathrm{D} 4$ and $\left\{x_{i}^{\prime}\right\}$ for $\mathrm{D} 4^{\prime}$ is

$$
\begin{aligned}
& x_{i}=x_{i}^{\prime} \cos \left(\theta_{i} \pi\right)+x_{i+4}^{\prime} \sin \left(\theta_{i} \pi\right), \\
& x_{i+4}=x_{i+4}^{\prime} \cos \left(\theta_{i} \pi\right)-x_{i}^{\prime} \sin \left(\theta_{i} \pi\right), \quad i=1, \ldots, 4, \\
& x_{9}=x_{9}^{\prime}+b .
\end{aligned}
$$

We see that the coordinates $x_{5, \cdots, 8}^{\prime}$ mix with $x_{1, \cdots, 4}$ with the coefficients $\sin \left(\theta_{i} \pi\right)$ and hence $F^{1234}$ in (15) gets the following contribution from $F^{5^{\prime} 6^{\prime} 7^{\prime} 8^{\prime}}$ :

$$
F_{(4)}^{1234}=\left(\prod_{i=1}^{4} \sin \left(\theta_{i} \pi\right)\right) F_{(4)}^{5^{\prime} 6^{\prime} 7^{\prime} 8^{\prime}}=-\frac{\mu_{\mathrm{D} 4^{\prime}}}{r^{4} \Omega_{4}} \frac{b}{r}\left(\prod_{i=1}^{4} \sin \left(\theta_{i} \pi\right)\right),
$$

where $r$ is the distance between $P$ and $Q$, and we have used eq. (17) and the fact that $P$ is away from $Q$ by $b$ in the direction of $x^{9}$. Let us take the origin on D4 at the nearest 
point from $\mathrm{D} 4^{\prime}$ and denote the coordinates of $\mathrm{Q}$ as $\left(y_{1}, y_{2}, y_{3}, y_{4}\right)$. Then $r=\mathrm{PQ}$ is given by $r^{2}=b^{2}+\sum_{i=1}^{4} y_{i}^{2} \sin ^{2}\left(\theta_{i} \pi\right)$. Using this expression and eq. (20), we obtain

$$
\begin{aligned}
& \mu_{\mathrm{D} 4} \int\left(\prod_{i=1}^{4} d y_{i}\right) F_{(4)}^{1234}=\mu_{\mathrm{D} 4} \int\left(\prod_{i=1}^{4} d y_{i}\right)\left(\prod_{i=1}^{4} \sin \left(\theta_{i} \pi\right)\right) F_{(4)}^{5^{\prime} 6^{\prime} 7^{\prime} 8^{\prime}} \\
& =-\mu_{\mathrm{D} 4} \int\left(\prod_{i=1}^{4} d y_{i}\right)\left(\prod_{i=1}^{4} \sin \left(\theta_{i} \pi\right)\right) \frac{\mu_{\mathrm{D} 4^{\prime}}}{r^{4} \Omega_{4}} \frac{b}{r}=-\frac{1}{2} \mu_{\mathrm{D} 4} \mu_{\mathrm{D} 4^{\prime}} \frac{b}{|b|}
\end{aligned}
$$

which is independent of the angles. Because $\mu_{\mathrm{D} 4}=\mu_{\mathrm{D} 4^{\prime}}=\left(\frac{1}{2 \pi \alpha^{\prime}}\right)^{\frac{1}{2}}$, eq. (21) turns out to be $-\frac{1}{4 \pi \alpha^{\prime}} \frac{b}{|b|}=-\frac{T_{0}}{2} \frac{b}{|b|}$, that is, one half contribution of the interaction between a fundamental string and a D-brane. This result is the same as the orthogonal case of [3].

Let us compare this result with our previous discussions on the potential. Consider the configuration $[\mathrm{A}]$. The above tension is the same as the force from the $\mathrm{R}(-1)^{F}$ term in (四) up to sign even if (5) is not satisfied. This means that the anomaly term corresponds to the $\mathrm{R}(-1)^{\mathrm{F}}$ term in the potential (四). so that the force is canceled because of unbroken supersymmetry [3, 23. This term may come from the effective action when we integrate the fermion with the lightest mass in supergravity. In fact the authors of [8] have derived the same term by doing so in M(atrix) theory.

To consider the configuration $[\mathrm{B}]$, we divide the one-loop potential of configuration [A] into two pieces as

$$
\mathrm{R}+\mathrm{NS}+\mathrm{NS}(-1)^{F}+\mathrm{R}(-1)^{F}=\left(\mathrm{R}+\mathrm{NS}+\mathrm{NS}(-1)^{F}-\mathrm{R}(-1)^{F}\right)+2 \mathrm{R}(-1)^{F} .
$$

The left hand side is the potential of configuration $[\mathrm{A}]$ which vanishes by supersymmetry. The first term on the right hand side is the potential for [B] which we computed in eq. (8) and the second term is for the created string. The double of the force induced by the anomaly term corresponds to that of the created string, which gives the same picture as in ref. [3].

String creation in type I' theory has also been discussed in refs. [4, 9, 12]. It should be straightforward to generalize our above discussions to similar configurations in such theory, and this will not be discussed in this paper.

\footnotetext{
${ }^{4}$ The overall sign is not significant.

${ }^{5}$ When one of the four angles is zero, both terms vanish, irrespectively of whether supersymmetry is unbroken or not.
} 
In summary, we have derived the results which are consistent with the creation of a fundamental string in the crossing process of D4-branes at angles and its variant con-

figuration by using the potentials of string and deformation of the anomaly term. It is consistent with the results of $\mathrm{M}$ (atrix) theory [8]. In the process, we have clarified how the configurations, and in particular, the fermionic zero-mode vacua change, giving a consistent picture of string creation. Our results also confirm the s-rule discussed for orthogonal case.

\section{Acknowledgments}

T.K. would like to thank the people of Particle Theory Group at Komaba for helpful comments, especially T. Yoneya for useful discussions. The work of N.O. and J.-G.Z. was supported in part by Grand-in-aid from the Ministry of Education, Science, Sports and Culture No. 96208.

\section{References}

[1] A. Hanany and E. Witten, Nucl. Phys. B492 (1997) 152, hep-th/9611230.

[2] C. Bachas, M. Douglas and M. Green, preprint, hep-th/9705074.

[3] U. Danielsson, G. Ferretti and I.R. Klebanov, Phys. Rev. Lett. 79 (1997) 1984, hepth/9705084; I.R. Klebanov, preprint, hep-th/9709160; U. Danielsson and G. Ferretti, preprint, hep-th/9709171.

[4] O. Bergman, M. Gaberdiel and G. Lifschytz, preprint, hep-th/9705130.

[5] S.P. de Alwis, Phys. Lett. B413 (1997) 49, hep-th/9706142.

[6] P.-M. Ho and Y.-S. Wu, preprint, hep-th/9708137.

[7] Y. Imamura, preprint, hep-th/9710026.

[8] N. Ohta, T. Shimizu and J.-G. Zhou, preprint, hep-th/9710218.

[9] O. Bergman, M.R. Gaberdiel and G. Lifschytz, preprint, hep-th/9711098. 
[10] T. Nakatsu, K. Ohta, T. Yokono and Y. Yoshida, preprint, hep-th/9711117.

[11] Y. Yoshida, preprint, hep-th/9711177.

[12] C.P. Bachas, M.B. Green and A. Schwimmer, preprint, hep-th/9712086.

[13] E. Bergshoeff, Y. Lozano and T. Ortin, preprint, hep-th/9712115.

[14] C.P. Bachas and M.B. Green, preprint, hep-th/9712187.

[15] M. Berkooz, M.R. Douglas and R.G. Leigh, Nucl. Phys. B480 (1996) 265, hepth/9606139.

[16] V. Balasubramanian and R.G. Leigh, Phys. Rev. D55 (1997) 6415, hep-th/9611165.

[17] J.P. Gauntlett, G.W. Gibbons, G. Papadopoulos and P.K. Townsend, Nucl. Phys. B500 (1997) 133, hep-th/9702202.

[18] P.K. Townsend, preprint, hep-th/9708074.

[19] H. Arfaei and M.M. Sheikh Jabbari, Phys. Lett. B394 (1997) 288, hep-th/9608167.

[20] N. Ohta and J.-G. Zhou, Phys. Lett. B418 (1998) 70, hep-th/9709065.

[21] M.M. Sheikh Jabbari, preprints, hep-th/9710121, hep-th/9608167.

[22] N. Ohta and P.K. Townsend, Phys. Lett. B418 (1998) 77, hep-th/9710129.

[23] G. Lifschytz, Phys. Lett. B388 (1996) 720, hep-th/9604156.

[24] A. Abouelsaood, C.G. Callan, C.R. Nappi and S.A. Yost, Nucl. Phys. B280 [FS18] (1987) 599.

[25] C.P. Bachas and M. Porrati, Phys. Lett. B296 (1992) 77, hep-th/9209032.

[26] M.B. Green and M. Gutperle, Nucl. Phys. B476 (1996) 484, hep-th/9604091.

[27] G. Lifschytz, Nucl. Phys. B499 (1997) 283, hep-th/9610125.

[28] H. Arfaei and M.M. Sheikh Jabbari, preprint, hep-th/9709054.

[29] M.M. Sheikh Jabbari, preprint, hep-th/9712199. 